\title{
Impact of Soil Degradation on Land Productivity of South El-Kalubia Governorate
}

\author{
Heba S. A. Rashed
}

Soil Department, Faculty of Agriculture, Moshtohor, Benha

University, Cairo, Egypt.

\begin{abstract}
DIRECT evaluation of land productive capacity was excuted $\mathcal{U}_{\text {depending on the parametric method for evaluation of land }}$ productivity in South El-Kalubia, showed that ten soil characteristics were considered as limiting factors in land productivity. These factors are; moisture $(\mathrm{H})$, drainage $(\mathrm{D})$, soil depth $(\mathrm{P})$, slope $(\mathrm{E})$, soil $\mathrm{pH}(\mathrm{N})$, soluble salt concentration (S), texture/structure $(\mathrm{T})$, organic matter $(\mathrm{O})$, cation exchange capacity (A) and mineral reserve (M). The study area which covers about 44150 ha $^{2} .4$ main geomorphologic units, i.e. flood plain, hummuky area, hilly area and turtle back. It is considered as unstable ecosystem due to the active degradation resulting from climate, relief and soil properties. The most active land degradation features are; salinization, sodification, waterlogging and compaction, all of which have negative impacts on land productivity. Soil topograph, physical and chemical properties too were measured to assess land productivity index (LPI). Rating of soil and topographic parameters were calculated using productivity formula for productivity classification for each land mapping unit. Most of the study areas 96.38\%; 36232 ha are excellent and good classes (class I and II) in terms of agricultural use. The remaining area $3.62 \% ; 1364$ ha are extremely poor class or nil (class V).
\end{abstract}

Keywords: Land degradation, Land productivity, El-Kalubia governorate.

Aagricultural productivity may be measured by what is termed "total factor productivity" (TFP). This method of calculating agricultural productivity compares an index of agricultural inputs to an index of outputs (Fuglie et al., 2007). Land productive capacity or land quality is a comprehension, at the same time a precise concept in terms of agricultural activities. It is defined as a measure of capability of land to perform specific functions (Devi and Kumar, 2008). Undoubtedly, one of the ways to provide food is to increase production per area and to use the land with respect to its potentiality in an appropriate way.

Soil fertility is its inherent capacity to supply crops grown on it with nutrients in adequate amounts and suitable proportions, whereas soil productivity is a wider term referring to the ability of a soil to yield crops. The chief factors in soil productivity are soil organic matter (including microbial biomass), soil texture, structure, depth, nutrient content, water-storage capacity, reaction and absence of toxic substances, all of which depend on physical, hydraulic, chemical and 
biologic characteristics (Dengiz, 2007). According to Pieri et al. (1995) and Dengiz et al. (2010) land quality is defined as "the condition and capacity of land, including its soil, climate, topography and biological properties, for purposes of production, conservation, and environmental management".

For many farmers (especially in non-industrial countries) agricultural productivity may mean much more. A productive farm is one that provides most of the resources necessary for the farmer's family to live, such as food, fuel, fiber, healing plants, ... etc. It is a farm which ensures food security as well as a way to sustain the well-being of a community. This implies that a productive farm is also one which is able to ensure proper management of natural resources, such as biodiversity, soil, water, ... etc. For most farmers, a productive farm would also produce more goods than required for the community in order to allow trade (Mundlak, 2007). Dengiz and Sağlam (2012) state that agriculture is one of the world's most important activities supporting human life. From the beginning of the civilization, man has used the land resources to satisfy his needs. Land resources regeneration is very slow while the population growth is very fast, leading to unbalances. Potential land use assessment is likely to be the prediction of land potential for productive land use types.

Land degradation is a process in which the value of the biophysical environment is negatively affected by a combination of human-induced processes acting upon the land. Environmental degradation is a gradual destruction or reduction of the quality and quantity of human activities, animal activities or natural means. It is viewed as a disturbance to the land perceived to be deleterious or undesirable. Natural hazards are excluded as a cause. However, human activities can indirectly cause hazards such as floods and bush fires. This is considered an important topic of the $21^{\text {st }}$ century due to the implications land degradation has upon agronomic productivity, the environment, and food security. It is estimated that up to $40 \%$ of the world's agricultural land is seriously degraded (Johnson and Lewis, 2007). Land degradation is defined by Bai et al. (2008) and Pierre (2010) as the long-term loss of ecosystem function and productivity caused by disturbances from which the land cannot recover unaided.

According to FAO (1994) there are six major causes of land degradation in the region, i.e. (1) deforestation, (2) shortage of land due to increased populations, (3) poor land use, (4) insecure land tenure, (5) inappropriate land management practices and (6) poverty. A major shortcoming of available statistics on land degradation is the lack of cause-effect relationship between severity of degradation and productivity. Criteria for designating different classes of land degradation are generally based on land properties rather than their impact on productivity. Assessing the productivity effects of land degradation is a challenging task (Eswaran et al., 2001). 


\section{Materials and Methods}

Location and geomorphology of the study area

El-Kalubia Governorate is located between longitudes $30^{\circ} 10^{\prime}$ and $30^{\circ} 40^{\prime} \mathrm{E}$ and latitudes $31^{\circ} 5^{\prime}$ and $31^{\circ} 25^{\prime} \mathrm{N}$, and bounded to the north by Dakahlia Governorate, to the south by Cairo and Giza Governorates, to the east by Sharkia Governorate and the west by Monoufiya Governorate. The total area of the Governorate is $94400 \mathrm{ha}^{2}$, and it represents about $0.1 \%$ of the total area of Egypt. Figure 1 shows a geomorphologic map of the South Kalubia area of the current study. This map is derived from images of the Landsat 7 satellite giving a map at a final scale of 1:250000 and by extracting raster geomorphologic units, victor geomorphologic units were then obtained using ARC GIS 9.4 software.

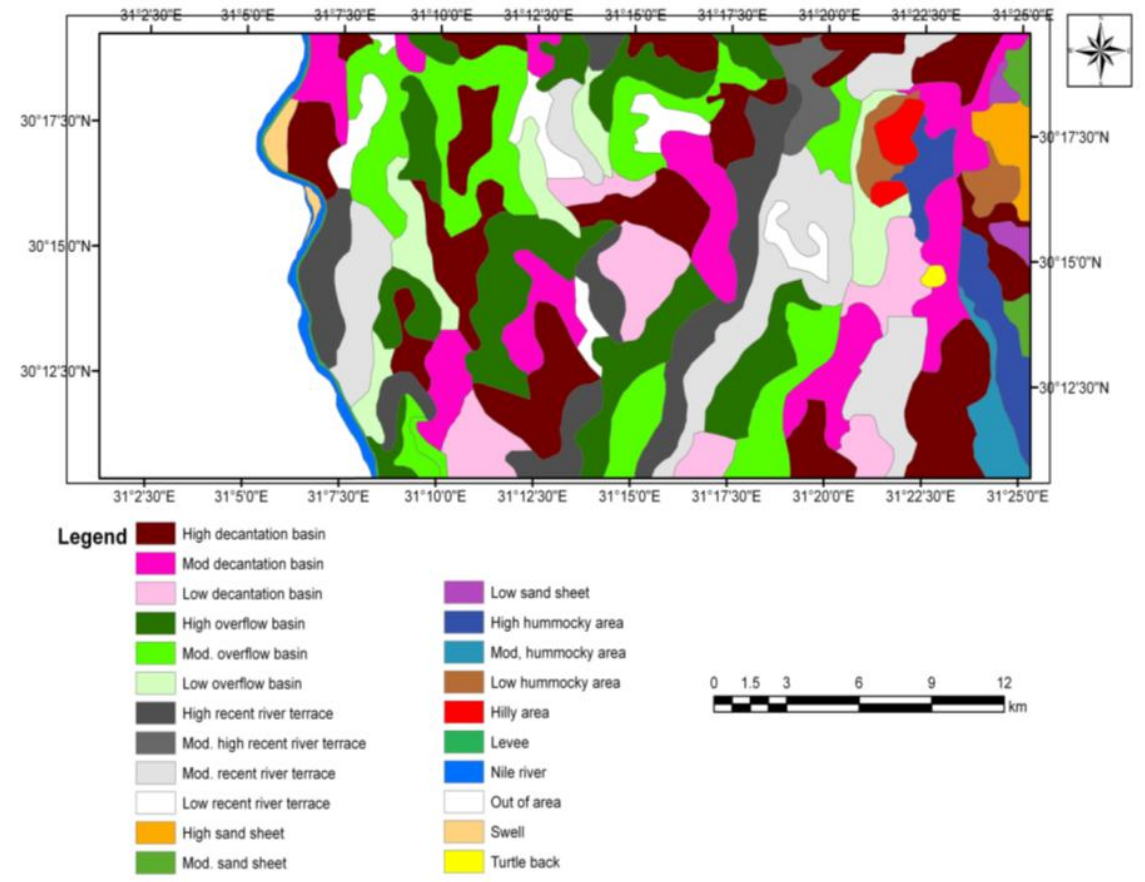

Fig.1. Geomorphology map of South El-Kalubia.

The Main geomorphic units and landforms of the study area

The geomorphic units of the study area were recognized four main landforms:

Flood plain (based on the relief, this is divided into the following three types a) Decantation basins, b) Overflow basins, C) Recent river terraces, d) Levee, and e) Swales), 2- Hummocky area, Hilly area and Turtle back (Zahra, 2007). Table 1 shows geomorphic units and their area. 
TABLE 1. Geomorphic units, landform, and the total area of the studied area.

\begin{tabular}{|l|l|c|}
\hline \multicolumn{1}{|c|}{ Geomorphic units } & \multicolumn{1}{|c|}{ Landform } & \% of Total area \\
\hline \multirow{4}{*}{ Flood plain } & Decantation basin (DB) & 25.43 \\
\cline { 2 - 3 } & Overflow basin (OB) & 38.72 \\
\cline { 2 - 3 } & Recent river terrace (RT) & 31.15 \\
\cline { 2 - 3 } & Levee (LV) & 0.45 \\
\cline { 2 - 3 } & Swale (SW) & 0.63 \\
\hline \multirow{2}{*}{ Hummocky area } & $(\mathrm{HM})$ & 2.26 \\
\hline \multirow{2}{*}{ Hilly area } & $(\mathrm{HL})$ & 1.19 \\
\hline Turtle back & & 0.17 \\
\hline
\end{tabular}

Site selection and soil characteristics

Based on distribution of physiographic units, 17 soil profiles were chosen to represent the studied area.

Detailed morphological description and classification of the selected soil profiles were recorded on the basis outlined by FAO (1990) and the USDA Soil Survey Staff (1999). Disturbed samples were air dried; ground gently, then sieved through $2 \mathrm{~mm}$ sieve. The soil samples were analyzed for particle size distribution, chemical and physical analyses (Rowell, 1995). Soil color in both wet and dry samples was determined using the Munssel color chart (Anon, 1975).

\section{Data analysis}

A parametric method for land evaluation has been proposed by Riquier et al. (1970) who stated that limitations are negative and complex concepts and that present and future capabilities are better expressed in terms of productivity. The system avoids economic and sociological considerations which lie outside the province of soil science. The system suggested the calculation of a productivity index considering ten factors as determining land productivity. They are moisture $(\mathrm{H})$, drainage $(\mathrm{D})$, soil depth $(\mathrm{P})$, slope $(\mathrm{E})$, soil $\mathrm{pH}(\mathrm{N})$, soluble salt concentration $(\mathrm{S})$, texture/structure $(\mathrm{T})$, organic matter $(\mathrm{O})$, cation exchange capacity (A) and mineral reserve (M). A mathematical formula expressing productivity resultant from those factors is as follows:

Land Productivity Index $(\mathrm{LPI})=\mathrm{H} / 100 \times \mathrm{D} / 100 \times \mathrm{P} / 100 \times \mathrm{E} / 100 \times \mathrm{N} / 100 \times$ $\mathrm{S} / 100 \times \mathrm{T} / 100 \times \mathrm{O} / 100 \times \mathrm{A} / 100 \times \mathrm{M} / 100$

Each factor is rated on a scale from 0 to 100 and the resultant index of productivity, lies between 0 and 100, and is set against a scale placing the soil in one of the following five productivity classes (Table 2). 
TABLE 2. Land productivity classes.

\begin{tabular}{|c|c|c|}
\hline Land Productivity Index & Definition & Class \\
\hline $0.65-1.00$ & Excellent & I \\
\hline $0.35-0.64$ & Good & II \\
\hline $0.20-0.34$ & Average & III \\
\hline $0.08-0.19$ & poor & IV \\
\hline $0.00-0.07$ & Extremely poor or nil & V \\
\hline
\end{tabular}

Each of the land characteristic with associated attribute data are digitally encoded in a GIS database to eventually generate ten thematic layers. The diagnostic factors of each thematic layer were assigned values of factor rating identified in Tables 3-7.

TABLE 3. Definition of soil moisture and organic matter.

\begin{tabular}{|c|c|c|c|}
\hline \multicolumn{2}{|r|}{ Soil moisture content $(\mathrm{H})$} & \multicolumn{2}{|r|}{ Organic matter in A1 horizon (O) } \\
\hline $\begin{array}{l}\mathbf{H} \\
\mathbf{1}\end{array}$ & $\begin{array}{l}\text { Rooting zone below wilting point all } \\
\text { the year round }\end{array}$ & O1 & $\begin{array}{l}\text { Very little organic matter, less than } \\
10 \mathrm{~g} / \mathrm{kg}\end{array}$ \\
\hline $\begin{array}{l}\mathbf{H} \\
\mathbf{2}\end{array}$ & $\begin{array}{l}\text { Rooting zone below wilting point } \\
\text { for } 9 \text { to } 11 \text { months of the year } \\
\text { H2a: } 11, \mathrm{H} 2 \mathrm{~b}: 10, \mathrm{H} 2 \mathrm{c}: 9 \text { months, }\end{array}$ & $\mathbf{O 2}$ & Little organic matter, $10-20 \mathrm{~g} / \mathrm{kg}$ \\
\hline $\begin{array}{l}\mathbf{H} \\
\mathbf{3}\end{array}$ & $\begin{array}{l}\text { Rooting zone below wilting point } \\
\text { for } 6 \text { to } 8 \text { months of the year } \\
\text { H3a: } 8, \mathrm{H} 3 \mathrm{~b}: 7, \mathrm{H} 3 \mathrm{c}: 6 \text { months, }\end{array}$ & $\mathbf{O 3}$ & $\begin{array}{l}\text { Average organic matter content, } \\
20-50 \mathrm{~g} / \mathrm{kg}\end{array}$ \\
\hline $\begin{array}{l}\mathbf{H} \\
4\end{array}$ & $\begin{array}{l}\text { Rooting zone below wilting point } \\
\text { for } 3 \text { to } 5 \text { months of the year } \\
\text { H4a:5, H4b: } 4, \mathrm{H} 4 \mathrm{c}: 3 \text { months, }\end{array}$ & $\mathbf{O 4}$ & $\begin{array}{l}\text { High organic matter content, over } \\
50 \mathrm{~g} / \mathrm{kg}\end{array}$ \\
\hline $\begin{array}{l}\mathbf{H} \\
\mathbf{5}\end{array}$ & $\begin{array}{l}\text { Rooting zone above wilting point } \\
\text { and below field capacity for most of } \\
\text { the year }\end{array}$ & $\mathbf{O 5}$ & $\begin{array}{l}\text { Very high content but } \mathrm{C} / \mathrm{N} \text { ratio is } \\
\text { over } 25\end{array}$ \\
\hline
\end{tabular}

TABLE 4. Definition of soil depth and slope.

\begin{tabular}{|l|l|l|l|}
\hline \multicolumn{2}{|c|}{ Soil depth $($ P) } & \multicolumn{2}{c|}{ Slope (E) } \\
\hline P1 & $\begin{array}{l}\text { Rock outcrops with no soil cover or } \\
\text { very shallow cover }\end{array}$ & E1 & Flat 0-2\% \\
\hline P2 & Very shallow soil, $<30 \mathrm{~cm}$ & E2 & Slightly 2-6\% \\
\hline P3 & Shallow soil, $30-60 \mathrm{~cm}$ & E3 & Moderately 6-12\% \\
\hline P4 & Fairly deep soil, $60-90 \mathrm{~cm}$ & E4 & High 12-20\% \\
\hline P5 & Deep soil $90-120 \mathrm{~cm}$ & E5 & Very high $20-30 \%$ \\
\hline P6 & Very deep soil $>120 \mathrm{~cm}$ & E6 & Steep 30\% + \\
\hline
\end{tabular}


TABLE 5. Definition of soil drainage and reserves weatherable mineral.

\begin{tabular}{|l|l|c|l|}
\hline \multicolumn{2}{|c|}{ Drainage (D) } & \multicolumn{2}{l|}{$\begin{array}{l}\text { Reserves of weatherable mineral in B } \\
\text { horizon (M) }\end{array}$} \\
\hline D1a & $\begin{array}{l}\text { Marked waterlogging, water table almost } \\
\text { reaches the surface all year round }\end{array}$ & M1 & Reserves very low to nil \\
\hline D1b & Soil flooded for 2 to 4 months of year & M2 & Reserves fair \\
\hline D2a & $\begin{array}{l}\text { Moderate waterlogging, water table being } \\
\text { sufficiently close to the surface to harm deep } \\
\text { rooting plants }\end{array}$ & M2a & $\begin{array}{l}\text { Minerals derived from sands, } \\
\text { sandy material or ironstone }\end{array}$ \\
\hline D2b & $\begin{array}{l}\text { Total waterlogging of profile for 8 days to 2 } \\
\text { months }\end{array}$ & M2b & $\begin{array}{l}\text { Minerals derived from acid } \\
\text { rock }\end{array}$ \\
\hline D3a & $\begin{array}{l}\text { Good drainage, water table sufficiently low } \\
\text { not to impede crop growing }\end{array}$ & M2c & $\begin{array}{l}\text { Minerals derived from basic or } \\
\text { calcareous rocks }\end{array}$ \\
\hline D3b & $\begin{array}{l}\text { Waterlogging for brief period (flooding), } \\
\text { less than 8 days each time. }\end{array}$ & M3 & Reserves large \\
\hline D4 & $\begin{array}{l}\text { Well drained soil, deep water table; no } \\
\text { waterlogging of soil profile }\end{array}$ & M3a & $\begin{array}{l}\text { Sands, sandy materials or } \\
\text { ironstone }\end{array}$ \\
\cline { 2 - 4 } & & M3b & Acid rock \\
\cline { 2 - 4 } & M3c & Basic or calcareous rocks \\
\hline
\end{tabular}

TABLE 6. Definition of soil texture and structure of root zone, $\mathrm{pH}$ of $\mathrm{A}$ horizon, soluble salt content and cation exchange capacity.

\begin{tabular}{|c|c|c|c|}
\hline \multicolumn{2}{|r|}{ Texture and structure of root zone (T) } & \multicolumn{2}{|r|}{ pH of A horizon (N) } \\
\hline T1 & Pebbly, stony or gravelly soil & N1 & $\mathrm{pH}: 3.5-4.5$ \\
\hline T1a & $\begin{array}{l}\text { Pebbly, stony or gravelly }>60 \% \text { by } \\
\text { weight }\end{array}$ & N2 & $\mathrm{pH}: 4.5-5.0$ \\
\hline T1b & $\begin{array}{l}\text { Pebbly, stony or gravelly from } 40 \text { to } \\
60 \%\end{array}$ & N3 & pH: $5.0-6.0$ \\
\hline T1c & Pebbly, stony from 20 to $40 \%$ & $\mathbf{N 4}$ & $\mathrm{pH}: 6.0-7.0$ \\
\hline $\mathbf{T 2}$ & Extremely coarse textured soil & \multirow[t]{2}{*}{ N5 } & \multirow[t]{2}{*}{$\mathrm{pH}: 7.0-8.5$} \\
\hline $\mathbf{T} 2 \mathbf{a}$ & Pure sand, of particle structure & & \\
\hline T2b & $\begin{array}{l}\text { Extremely coarse textured soil (> 45\% } \\
\text { coarse sand) }\end{array}$ & \multicolumn{2}{|c|}{ Soluble salt content $(S)$} \\
\hline T2c & $\begin{array}{l}\text { Soil with non-decomposed raw humus } \\
\text { (> } 30 \% \text { organic matter) and fibrous } \\
\text { structure }\end{array}$ & S1 & $<0.2 \%$ \\
\hline T3 & $\begin{array}{l}\text { Dispersed clay of unstable structure } \\
(\mathrm{ESP}>15 \%)\end{array}$ & S2 & $0.2-0.4 \%$ \\
\hline T4 & $\begin{array}{l}\text { Light textured soil, fS, LS, SL, cS and } \\
\mathrm{Si}\end{array}$ & $\mathbf{S 3}$ & $0.4-0.6 \%$ \\
\hline T4a & Unstable structure & S4 & $0.6-0.8 \%$ \\
\hline T4b & Stable structure & S5 & $0.8-1.0 \%$ \\
\hline T5 & Heavy-textured soil: $\mathrm{C}$ or $\mathrm{SiC}$ & S6 & $>1.0 \%$ \\
\hline T5a & Massive to large prismatic structure & S7 & $\begin{array}{l}\text { Total soluble salt (including } \mathrm{Na} 2 \mathrm{CO} 3 \text { ) } \\
0.1-0.3 \%\end{array}$ \\
\hline T5b & $\begin{array}{l}\text { Angular to crumb structure or massive } \\
\text { but highly porous }\end{array}$ & S8 & $0.3-0.6 \%$ \\
\hline T6 & $\begin{array}{l}\text { Medium-heavy soil: heavy SL, SC, } \\
\text { CL, SiCL, Si }\end{array}$ & S9 & $>0.6 \%$ \\
\hline T6a & Massive to large prismatic structure & \multicolumn{2}{|c|}{ Cation Exchange Capacity (A) } \\
\hline \multirow[t]{2}{*}{ T6b } & \multirow[t]{2}{*}{$\begin{array}{l}\text { Angular to crumb structure (massive } \\
\text { but porous }\end{array}$} & A0 & $\begin{array}{l}\begin{array}{l}\text { Exchange capacity of clay } \\
\mathrm{cmol}_{\mathrm{c}} / \mathrm{kg}\end{array} \\
\end{array}$ \\
\hline & & A1 & $\begin{array}{l}\begin{array}{l}\text { Exchange capacity of clay } \\
\mathrm{cmol}_{\mathrm{c}} / \mathrm{kg} \\
\text { sesquioxides) }\end{array} \\
\text { (probably kaolinite }\end{array}$ \\
\hline \multirow[t]{2}{*}{ T7 } & \multirow[t]{2}{*}{$\begin{array}{l}\text { Soil of average, balanced texture: L, } \\
\text { SiL and SCL }\end{array}$} & $\mathbf{A 2}$ & $\begin{array}{l}\text { Exchange capacity of clay from } 20 \text { to } \\
40 \mathrm{cmol}_{\mathrm{c}} / \mathrm{kg}\end{array}$ \\
\hline & & $\mathbf{A 3}$ & Exchange capacity of clay $>40 \mathrm{cmol}_{d} / \mathrm{kg}$ \\
\hline
\end{tabular}

Note: fS: fine sand, LS: loamy sand, SL: sandy loam, S: sand, C: clay, Si: Silt, SiC: silty clay, cS: coarse sand

Egypt. J. Soil Sci. 55, No. 1 (2015) 
IMPACT OF SOIL DEGRADATION ON LAND PRODUCTIVITY...

TABLE 7. Ratings of different soil and land characteristics.

\begin{tabular}{|c|c|c|c|c|c|c|c|c|}
\hline \multicolumn{2}{|c|}{ Factor } & \multicolumn{3}{|c|}{ Crop growing } & \multicolumn{2}{|c|}{ Factor } & \multicolumn{2}{|r|}{ Crop growing } \\
\hline \multicolumn{5}{|c|}{ 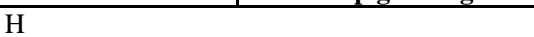 } & \multicolumn{4}{|l|}{$\mathrm{O}$} \\
\hline \multicolumn{2}{|l|}{ H1 } & \multicolumn{3}{|l|}{5} & \multirow{3}{*}{\multicolumn{2}{|c|}{$\mathrm{O} 1$}} & \multirow{3}{*}{\multicolumn{2}{|c|}{85}} \\
\hline \multicolumn{2}{|l|}{$\mathrm{H} 2 \mathrm{a}$} & \multicolumn{3}{|l|}{10} & & & & \\
\hline \multicolumn{2}{|l|}{$\mathrm{H} 2 \mathrm{~b}$} & \multicolumn{3}{|l|}{20} & & & & \\
\hline $\mathrm{H} 2 \mathrm{c}$ & & \multicolumn{3}{|l|}{40} & \multirow{3}{*}{\multicolumn{2}{|c|}{$\mathrm{O} 2$}} & \multirow{3}{*}{\multicolumn{2}{|c|}{90}} \\
\hline \multicolumn{2}{|l|}{$\mathrm{H} 3 \mathrm{a}$} & \multicolumn{3}{|l|}{50} & & & & \\
\hline $\mathrm{H} 3 \mathrm{~b}$ & & 60 & & & & & & \\
\hline $\mathrm{H} 3 \mathrm{c}$ & & 70 & & & $\mathrm{O} 3$ & & 100 & \\
\hline $\mathrm{H} 4 \mathrm{a}$ & & 80 & & & $\mathrm{O} 4$ & & 100 & \\
\hline $\mathrm{H} 4 \mathrm{~b}$ & & 90 & & & & & & \\
\hline $\mathrm{H} 4 \mathrm{c}$ & & 100 & & & O5 & & 70 & \\
\hline $\mathrm{H} 5$ & & 100 & & & & & & \\
\hline $\mathrm{P}$ & & & & & $E$ & & & \\
\hline $\mathrm{P} 1$ & & 5 & & & E1 & & & 100 \\
\hline $\mathrm{P} 2$ & & 20 & & & E2 & & & 95 \\
\hline P3 & & 50 & & & E3 & & & 90 \\
\hline $\mathrm{P} 4$ & & 80 & & & E4 & & & 85 \\
\hline P5 & & 100 & & & E5 & & & 80 \\
\hline P6 & & 100 & & & E6 & & & 80 \\
\hline $\mathrm{D}$ & $\mathrm{H} 4, \mathrm{H} 5$ & $\mathrm{H} 2, \mathrm{H}$ & & & $\mathrm{M}$ & $\mathrm{H} 1, \mathrm{H} 2, \mathrm{H} 3$ & & H4,H5 \\
\hline D1 & 10 & 40 & & & M1 & 85 & & 85 \\
\hline & & & & & M2a & 85 & & 90 \\
\hline D2 & 40 & 80 & & & $\mathrm{M} 2 \mathrm{~b}$ & 90 & & 95 \\
\hline & & & & & M2c & 95 & & 100 \\
\hline D3 & 80 & 90 & & & M3a & 90 & & 95 \\
\hline & & & & & M3b & 95 & & 100 \\
\hline D4 & 100 & 100 & & & M3c & 100 & & 100 \\
\hline $\mathrm{T}$ & & & & & & $\mathrm{N}$ & & \\
\hline T1a & & 10 & & & N1 & 40 & & \\
\hline T1b & & 30 & & & N2 & 50 & & \\
\hline T1c & & 60 & & & N3 & 60 & & \\
\hline & & $\mathrm{H} 4,5,6$ & $\mathrm{H} 3$ & $\mathrm{H} 1,2$ & N4 & 80 & & \\
\hline $\mathrm{T} 2 \mathrm{a}$ & & 10 & 10 & 10 & & & & \\
\hline & & & & & N5 & 100 & & \\
\hline $\mathrm{T} 2 \mathrm{~b}$ & & 30 & 20 & 10 & & & & \\
\hline & & & & & $\mathrm{S}$ & $\mathrm{T} 1, \mathrm{~T} 2, \mathrm{~T} 4$ & & $\mathrm{~T} 5, \mathrm{~T} 6, \mathrm{~T} 7$ \\
\hline $\mathrm{T} 2 \mathrm{c}$ & & 30 & 30 & 30 & S1 & 100 & & 100 \\
\hline & & & & & $\mathrm{S} 2$ & 70 & & 90 \\
\hline T3 & & 30 & 20 & 10 & S3 & 50 & & 80 \\
\hline & & & & & S4 & 25 & & 40 \\
\hline T4a & & 40 & 30 & 30 & S5 & 15 & & 25 \\
\hline & & & & & S6 & 5 & & 15 \\
\hline T4b & & 50 & 50 & 60 & S7 & 60 & & 90 \\
\hline & & & & & S8 & 15 & & 60 \\
\hline T5a & & 50 & 60 & 20 & S9 & 5 & & 15 \\
\hline & & & & & $\mathrm{A}$ & & & \\
\hline T5b & & 80 & 80 & 60 & $\mathrm{~A} 0$ & & 85 & \\
\hline T6a & & 80 & 80 & 60 & A1 & & 90 & \\
\hline T6b & & 90 & 90 & 90 & $\mathrm{~A} 2$ & & 95 & \\
\hline & & & & & A3 & & 100 & \\
\hline T7 & & 100 & 100 & 100 & & & & \\
\hline
\end{tabular}

Egypt. J. Soil Sci. 55, No.1 (2015) 


\section{Results and Discussion}

Soil degradation processes

The main types of human induced land degradation in the investigated areas are salinization, sodification (alkalinization), soil compaction and water-logging. These types are affected by the human activities as follows:

In the southern part of Kalubia Governorate, there are many land degradation processes. Salinization and sodification (alkalinization) are due to accumulation of excess salts in the root zone resulting in partial or complete loss of soil productivity. The reason of salinization and sodification in the area may be poor irrigation and drainage management or high evapo-transpiration. A high salt content of the irrigation water or lack of attention given to drainage would lead to rapid salinization and / or sodification. This type of salt accumulation mainly occurs under arid and semi-arid conditions. Compaction is mainly shown as massive structure and low stability of structure under improper human activities. In the studied areas soil compaction seemed to be caused by improper use of heavy machinery, shortage of the fallow period and the excessive use of chemical fertilizers. Water-logging is one of the factors responsible for soil salinity. Over irrigation, insufficient drainage and destruction of subsurface drainage networks are main causes of water-logging in the area.

Soil characteristics and degradation evidences

Table 8 illustrates soil properties and degradation evidences of the study area.

TABLE 8. Soil properties / degradation evidences.

\begin{tabular}{|l|c|c|c|c|}
\hline \multirow{2}{*}{$\begin{array}{l}\text { Mapping } \\
\text { unit }\end{array}$} & \multicolumn{2}{|c|}{ Chemical degradation } & \multicolumn{2}{c|}{ Physical degradation } \\
\cline { 2 - 5 } & Salinity (s) & Sodacity (a) & Compaction (c) & $\begin{array}{c}\text { Water logging } \\
\text { (w) }\end{array}$ \\
\hline DB & 1.75 & 3.03 & 1.26 & 150 \\
\hline OB & 2.58 & 7.15 & 1.32 & 150 \\
\hline RT & 4.93 & 3.07 & 1.22 & 140 \\
\hline LV & 1.23 & 5.78 & 1.27 & 150 \\
\hline SW & 2.06 & 8.85 & 1.20 & 120 \\
\hline HM & 3.05 & 4.35 & 1.54 & 110 \\
\hline HL & 6.13 & 11.65 & 1.62 & 95 \\
\hline TB & 2.17 & 2.70 & 1.68 & 80 \\
\hline
\end{tabular}

\section{FAO/UNEP criteria}

The criteria (FAO/UNEP, 1978) are used to determine the degree, class and rate of different types belonging to land degradation as shown as in Table 9.

Egypt. J. Soil Sci. 55, No. 1 (2015) 
IMPACT OF SOIL DEGRADATION ON LAND PRODUCTIVITY...

TABLE 9. Criteria used to determine the degree of different types of degradation.

\begin{tabular}{|c|c|c|c|c|c|c|c|}
\hline $\begin{array}{l}\text { Criteria/degra } \\
\text { dation type }\end{array}$ & Indicator & Unit & 1 & 2 & 3 & 4 & 5 \\
\hline \multirow[t]{2}{*}{ Salinization } & \multirow[t]{2}{*}{ EC } & $\mathrm{dS} / \mathrm{m}$ & $<4$ & $4-8$ & $8-16$ & $16-32$ & $>32$ \\
\hline & & Class & None & Slight & Moderate & Severe & $\begin{array}{c}\text { Very } \\
\text { severe }\end{array}$ \\
\hline \multirow[t]{2}{*}{ Sodicity } & \multirow[t]{2}{*}{ ESP } & $\%$ & $<10$ & $10-15$ & $15-30$ & $30-50$ & $>50$ \\
\hline & & Class & None & Slight & Moderate & Severe & $\begin{array}{c}\text { Very } \\
\text { severe }\end{array}$ \\
\hline \multirow[t]{2}{*}{ Compaction } & \multirow{2}{*}{$\begin{array}{c}\text { Bulk } \\
\text { density }\end{array}$} & $\mathrm{g} / \mathrm{cm}^{3}$ & $>1.8$ & 1.6-1.8 & 1.4-1.6 & $1.2-1.4$ & $<1.2$ \\
\hline & & Class & losses & $\begin{array}{c}\text { Slightly } \\
\text { hard }\end{array}$ & Hard & Very hard & $\begin{array}{c}\text { Extremely } \\
\text { hard }\end{array}$ \\
\hline \multirow[t]{2}{*}{ Waterlogging } & \multirow{2}{*}{$\begin{array}{l}\text { Water } \\
\text { logging }\end{array}$} & $\mathbf{c m}$ & $>150$ & $150-100$ & $100-50$ & $50-30$ & $<30$ \\
\hline & & Class & None & Slight & Moderate & Severe & $\begin{array}{c}\text { Very } \\
\text { severe } \\
\end{array}$ \\
\hline
\end{tabular}

Notice: 1-5 means degree of hazard. $\quad$ Source: After FAO/UNEP (1978).

\section{Land degradation assessment}

With regard to salinity hazards, soils of DB, OB, LV, SW, HM and TB belong to class 1 (non-saline), while the others belong generally to class 2 (slight). With regard to sodicity hazards, all soils belong to class 1 (none) except for HL belongs to class 2 (slight). As for compaction hazards, soils of HL and TB belong to class 2 (slightly hard), whereas soils of DB, OB, RT, LV and SW belong to class 4 (very hard), except for HM belongs to class 3 (hard). All soils of the studied area belong to class 2 (slight) regarding water logging hazards, except for HL and TB belong to class 3 (moderate).

Table 10 shows the summary of land degradation assessment for soils of the study area.

TABLE 10. Land degradation assessment.

\begin{tabular}{|c|c|c|c|c|}
\hline \multirow{2}{*}{$\begin{array}{c}\text { Mapping } \\
\text { unit }\end{array}$} & $\begin{array}{c}\text { Salinity (a) } \\
\text { degree }\end{array}$ & $\begin{array}{c}\text { Sodicity (a) } \\
\text { degree }\end{array}$ & $\begin{array}{c}\text { Compaction (c) } \\
\text { degree }\end{array}$ & $\begin{array}{c}\text { Water logging } \\
\text { (w) degree }\end{array}$ \\
\cline { 2 - 5 } & N(class 1) & N(class 1) & V(class 4) & S(class 2) \\
\hline OB & N(class 1) & N(class 1) & V(class 4) & S(class 2) \\
\hline RT & S(class 2) & N(class 1) & V(class 4) & S(class 2) \\
\hline LV & N(class 1) & N(class 1) & V(class 4) & S(class 2) \\
\hline SW & N(class 1) & N(class 1) & V(class 4) & S(class 2) \\
\hline HM & N(class 1) & N(class 1) & H(class 3) & S(class 2) \\
\hline HL & S(class 2) & S(class 2) & S(class 2) & M(class 3) \\
\hline TB & N(class 1) & N(class 1) & S(class 2) & M(class 3) \\
\hline
\end{tabular}

Notes: $\mathrm{N}=$ None, $\mathrm{S}=$ Slight, $\mathrm{M}=$ Moderate, $\mathrm{V}=$ Very hard, $\mathrm{H}=$ Hard. 


\section{Land productivity}

Land productivity is assessed using the productivity model after Riquier et al. (1970). Land productivity classification groups are distinguished in precise numerical units. Classifications, which meet soil productivity requirements, would be taken as the highest grades. Soils with extreme limitations would be the lowest ones. Intermediate grades would be placed in between the two extreme conditions. Values of the factors of land productivity are shown in Table 11. Soil characteristics relevant to productivity are shown in Table 12, while assessment of soil productivity could be obtained by matching soil characteristics with its counterpart of the requires model rating as shown in Table 13.

TABLE 11. Values of the factors of land productivity of the studied soils of the investigated area.

\begin{tabular}{|c|c|c|c|c|c|c|c|c|c|c|}
\hline 昭 & 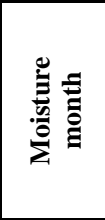 & 总 & 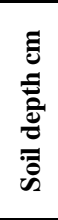 & $\frac{\check{0}}{\pi}$ & $\frac{\pi}{2}$ & 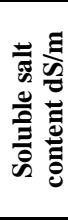 & 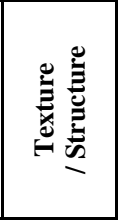 & 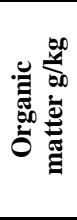 & 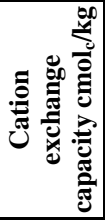 & 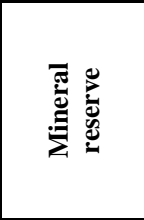 \\
\hline DB & 9 month & $\begin{array}{c}\text { Well } \\
\text { drained }\end{array}$ & 150 & 1.0 & 7.1 & 1.75 & Clay & 18.2 & 55.6 & $\begin{array}{c}\text { from sands, } \\
\text { sandy } \\
\text { material or } \\
\text { ironstone }\end{array}$ \\
\hline OB & 9 month & $\begin{array}{c}\text { Well } \\
\text { drained }\end{array}$ & 150 & 1.0 & 7.4 & 2.58 & Clay loam & 14.6 & 49.3 & $\begin{array}{c}\text { from sands, } \\
\text { sandy } \\
\text { material or } \\
\text { ironstone }\end{array}$ \\
\hline RT & 9 month & $\begin{array}{c}\text { Good } \\
\text { drained }\end{array}$ & 140 & 1.0 & 7.4 & 4.93 & Clay & 15.4 & 50.8 & $\begin{array}{c}\text { from sands, } \\
\text { sandy } \\
\text { material or } \\
\text { ironstone } \\
\end{array}$ \\
\hline $\mathbf{L V}$ & 9 month & $\begin{array}{c}\text { Good } \\
\text { drained }\end{array}$ & 150 & 1.0 & 8.2 & 1.23 & Clay & 16.7 & 57.1 & \begin{tabular}{|c|} 
from sands, \\
sandy \\
material or \\
ironstone \\
\end{tabular} \\
\hline SW & 9 month & $\begin{array}{c}\text { Well } \\
\text { drained }\end{array}$ & 120 & 1.0 & 7.8 & 2.06 & Clay & 15.9 & 50.9 & \begin{tabular}{|c|} 
from sands, \\
sandy \\
material or \\
ironstone
\end{tabular} \\
\hline HM & 9 month & $\begin{array}{c}\text { Good } \\
\text { drained }\end{array}$ & 110 & 1.5 & 7.5 & 3.05 & $\begin{array}{c}\text { Sandy } \\
\text { loam }\end{array}$ & 6.5 & 16.5 & $\begin{array}{c}\text { from sands, } \\
\text { sandy } \\
\text { material or } \\
\text { ironstone }\end{array}$ \\
\hline HL & 9 month & $\begin{array}{c}\text { Good } \\
\text { drained }\end{array}$ & 95 & 1.5 & 8.0 & 6.13 & Sand & 7.3 & 12.1 & \begin{tabular}{|c|} 
from sands, \\
sandy \\
material or \\
ironstone \\
\end{tabular} \\
\hline TB & 9 month & $\begin{array}{c}\text { Good } \\
\text { drained }\end{array}$ & 80 & 2.0 & 7.5 & 2.17 & Sand & 5.6 & 3.6 & $\begin{array}{c}\text { from sands, } \\
\text { sandy } \\
\text { material or } \\
\text { ironstone }\end{array}$ \\
\hline
\end{tabular}

Egypt. J. Soil Sci. 55, No. 1 (2015) 
TABLE 12. Soil characteristics of the investigated area.

\begin{tabular}{|c|c|c|c|c|c|c|c|c|c|c|}
\hline 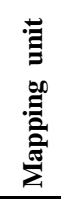 & 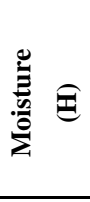 & 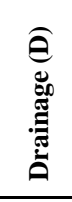 & 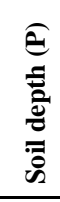 & $\begin{array}{l}\text { 됭 } \\
\text { ڤั }\end{array}$ & $\begin{array}{l}\widehat{z} \\
\bar{z} \\
\bar{z} \\
\overline{0} \\
\bar{n}\end{array}$ & 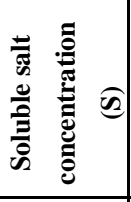 & 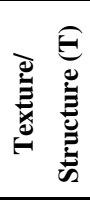 & 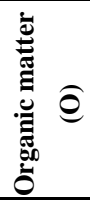 & 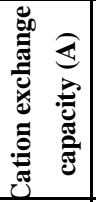 & 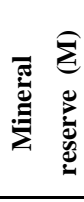 \\
\hline DB & $\mathrm{H} 4 \mathrm{c}$ & D4 & P6 & E1 & N5 & S1 & $\mathrm{T} 5 \mathrm{~b}$ & $\mathrm{O} 2$ & $\mathrm{~A} 3$ & M2c \\
\hline OB & $\mathrm{H} 4 \mathrm{c}$ & D4 & P6 & E1 & N5 & S1 & T6a & $\mathrm{O} 2$ & $\mathrm{~A} 3$ & M2c \\
\hline RT & $\mathrm{H} 4 \mathrm{c}$ & D3a & P6 & E1 & $\mathrm{N} 4$ & $\mathrm{~S} 2$ & $\mathrm{~T} 5 \mathrm{~b}$ & $\mathrm{O} 2$ & $\mathrm{~A} 3$ & M2c \\
\hline $\mathbf{L V}$ & $\mathrm{H} 4 \mathrm{c}$ & $\mathrm{D} 3 \mathrm{a}$ & P6 & E1 & $\mathrm{N} 4$ & S1 & $\mathrm{T} 5 \mathrm{~b}$ & $\mathrm{O} 2$ & $\mathrm{~A} 3$ & M2c \\
\hline SW & $\mathrm{H} 4 \mathrm{a}$ & D4 & P6 & E1 & N5 & S1 & $\mathrm{T} 5 \mathrm{~b}$ & $\mathrm{O} 2$ & $\mathrm{~A} 3$ & M2c \\
\hline HM & $\mathrm{H} 2 \mathrm{c}$ & D3a & P5 & E1 & N5 & $\mathrm{S} 1$ & $\mathrm{~T} 4 \mathrm{a}$ & $\mathrm{O} 1$ & $\mathrm{~A} 1$ & M2a \\
\hline HL & $\mathrm{H} 2 \mathrm{c}$ & D3a & P5 & E1 & N5 & S2 & $\mathrm{T} 2 \mathrm{a}$ & $\mathrm{O} 1$ & A1 & M2a \\
\hline TB & $\mathrm{H} 2 \mathrm{c}$ & D3a & $\mathrm{P} 4$ & E1 & N5 & S1 & $\mathrm{T} 2 \mathrm{a}$ & $\mathrm{O} 1$ & $\mathrm{~A} 0$ & M2a \\
\hline
\end{tabular}

Appreviation according to Riquier et al. (1970).

TABLE 13. Assessment of soil productivity of the investigated area.

\begin{tabular}{|c|c|c|c|c|c|c|c|c|c|c|c|c|}
\hline $\begin{array}{c}\text { Mapping } \\
\text { unit }\end{array}$ & $\mathbf{H}$ & $\mathbf{D}$ & $\mathbf{P}$ & $\mathbf{E}$ & $\mathbf{N}$ & $\mathbf{S}$ & $\mathbf{T}$ & $\mathbf{O}$ & $\mathbf{A}$ & $\mathbf{M}$ & $\begin{array}{c}\text { Land } \\
\text { Productivity } \\
\text { Index (LPI) }\end{array}$ & Class \\
\hline DB & 100 & 100 & 100 & 100 & 100 & 100 & 80 & 90 & 100 & 100 & 0.720 & I \\
\hline OB & 100 & 100 & 100 & 100 & 100 & 100 & 80 & 90 & 100 & 100 & 0.720 & I \\
\hline RT & 100 & 80 & 100 & 100 & 80 & 90 & 80 & 90 & 100 & 100 & 0.415 & II \\
\hline LV & 100 & 80 & 100 & 100 & 80 & 100 & 80 & 90 & 100 & 100 & 0.461 & II \\
\hline SW & 80 & 100 & 100 & 100 & 100 & 100 & 80 & 90 & 100 & 100 & 0.576 & II \\
\hline HM & 40 & 90 & 100 & 100 & 100 & 100 & 30 & 85 & 90 & 85 & 0.070 & V \\
\hline HL & 40 & 90 & 100 & 100 & 100 & 70 & 10 & 85 & 90 & 85 & 0.016 & V \\
\hline TB & 40 & 90 & 80 & 100 & 100 & 100 & 10 & 85 & 85 & 85 & 0.017 & V \\
\hline
\end{tabular}

Soil characteristics productivity rating, land productivity index and grades are shown in Tables 12 and 13.

\section{Land productivity assessment of the flood plain}

The mapping units in this landform could be grouped in two productivity grades as follows; mapping units DB and OB which have productivity grade I and LPI value of 0.720 . These units have $64.15 \%$ (24112 ha.), while RT, LV and SW have productivity grade II and LPI ranging between 0.415 and 0.576 , the percentage of these units is $32.23 \%$ (12120 ha.). The main limiting factors are moisture, drainage, texture/structure, salinity, $\mathrm{pH}$, organic matter and mineral reserve. 
Land productivity assessment of the hummocky area

Mapping unit HM has the productivity grade $\mathrm{v}$ and value of (LPI) 0.070 . This unit has grade $\mathrm{v}$ which represents $2.26 \%$ (850 ha.). The main limiting factors are moisture, drainage, texture/structure, organic matter, cation exchange capacity and mineral reserve

Land productivity assessment of the hilly area

Mapping unit HL has the productivity grade $\mathrm{v}$ and value of (LPI) 0.016, which represent $1.19 \%$ (447 ha.). The main limiting factors are moisture, drainage, texture/structure, salinity, organic matter, cation exchange capacity and mineral reserve.

Land productivity assessment of the turtle back

Mapping unit TB has the productivity grade $\mathrm{v}$ and value of (LPI) 0.017 , which represent $0.17 \%$ (67 ha.). The main limiting factors are moisture, drainage, soil depth, texture/structure, organic matter, cation exchange capacity and mineral reserve.

\section{Conclusions}

Achieving and maintaining good land quality are essential for sustainable agricultural production in an economically viable and environmentally safe manner. The goal of the current study is classifying land productivity to different categories, each of which corresponding to a certain level of details. At each level the interpretation differs in precision, objectives, requirements and assumptions. These successive steps help users for a better understanding of the system. Next to this study, more research should be devoted to these important topics, in particular validation of usefulness of LPI in decision making and implantation. The similar research should be also conducted for different soil types and environments.

Acknowledgment : The author would like to express deepest thanks to Prof. Dr. Ali Ahmed Abdel-Salam, Prof. of Soil Sci., Fac. Agric., Moshtohor, Benha University, for his guidance and comments during this work. Sincere thanks are also due to Prof. Dr. Hassan Hamza Abbas, Prof. of Soil Sci., Fac. Agric., Moshtohor, Benha University, for his comments during this work.

\section{Referances}

Anon (1975) Munsell Color Charts. Macbeth Division of Kollmorgen Corporation, 2441 North Cavert Street, Batimore, Maryland, USA.

Bai, Z.G., Dent, D.L., Olsson, L. and Schaepman, M.E. (2008) Global assessment of land degradation and improvement 1: Identification by Remote Sensing. Report 2008/01, FAO/ISRIC - Rome/Wageningen.

Dengiz, O. (2007) Assessment of soil productivity and erosion status for the AnkaraSogulca catchments using GIS. Int. J. Soil Sci. 2, 15-28.

Egypt. J. Soil Sci. 55, No. 1 (2015) 
Dengiz, O., Ozcan, H., Köksal, E.S., Baskan, O. and Kosker, Y. (2010) Sustainable natural resource management and environmental assessment in the salt lake (Tuz Golu) specially protected area. J. Environ. Mon. and Assess. 161, 327-342.

Dengiz, O. and Sağlam, M. (2012) Determination of land productivity index based on parametric approach using GIS technique. Eurasian Journal of Soil Science, 1, 51 - 57.

Devi, G. and Kumar, M.G. (2008) Remote sensing and GIS application for land quality assessment for coffee growing areas of Karnataka. J. Indian Soc. of Remote Sensing, 36, 89-97.

Eswaran, H., Lal, R. and Reich, P.F. (2001) Land Degradation: An Overview. Oxford Press. New Delhi, India.

FAO. (1990) Guidelines for Soil Profile Description. FAO, Rome, Italy.

FAO. (1994) Land degradation in South Asia: its severity, causes and effects upon the people, FAO, Rome, Italy.

FAO/UNEP. (1978) Methodology for assessing soil degradation. FAO, Rome, Italy.

Fuglie, K.O., James, M. and Eldon, B. (2007) Productivity growth in U.S. agriculture. EB-9, U.S. Dept. of Agriculture (USDA), Econ. Res. Serv. USA.

Johnson, D.L. and Lewis, L.A. (2007) Land Degradation: Creation and Destruction, Maryland, USA.

Mundlak, Y. (2007) Agricultural productivity and economic policies: Concepts and measurements, OECD Working Paper No. 75, OECD Development Center, August 1992, SourceOECD.org, 13 July 2007

Pieri, C., Dumanski, J., Hamblin, A. and Young, A. (1995) Land quality indicators. World Bank Discussion Papers 315. Washington, USA.

Pierre, B. (2010) A land degradation assessment and mapping method. A standard guideline proposal. Les dossiers thématiques du CSFD. CSFD/Agropolis International, Montpellier, France.

Riquier, J., Bramoa, D.L. and Cornet, J.P. (1970) A new system of appraisal in terms of actual and potential productivity. Soil Resources, Development and Conservation Service, Land and Water Development Division. FAO, Rome.

Rowell, D.L. (1995) Soil Science Methods and Applications. Library of Congress, USA.

Soil Survey Staff (1999) Soil taxonomy. A Basic System of Soil Classification for Making and Interpreting Surveys, Second edition, Agriculture Hand Book No. 436. USDA, USA..

Zahra, W.R.A. (2007) Evaluation of soil productivity using remote sensing and GIS techniques in some Kalubia soils. M.Sc. Thesis, Fac. of Agric., Moshtohor, Benh Univ., Egypt. 


\title{
تاثير تدهور التربة على انتاجية الاراضى جنوب محافظة القليوبية
}

\author{
هبة شوقى عبدالله راشد \\ قسم الار اضىـ كلية الزر اعةــ مشتهر - جامعة بنها- مصر.
}

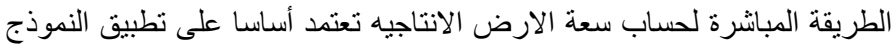

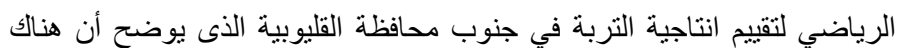

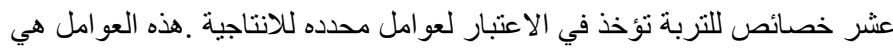

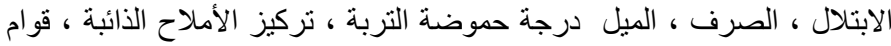

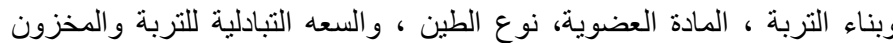

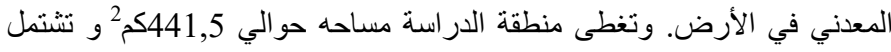
على أربع وحدات جيومورفولوجية وهئ الارضى السهل الفيضي ، منطقة الآكام ، منطقة

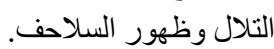

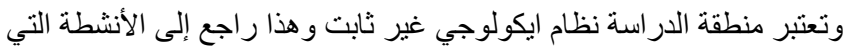

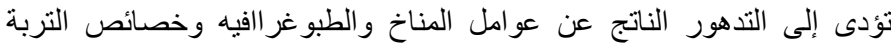

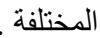

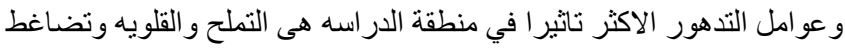

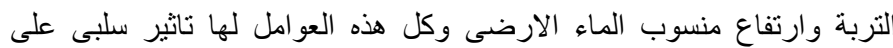

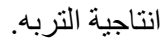

ومن خلال التحليلات و التقييمات للخواص: الطبو غر افية والطبيعية و الكيميائية

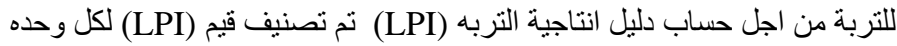

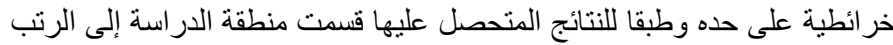

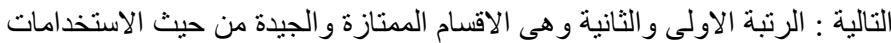

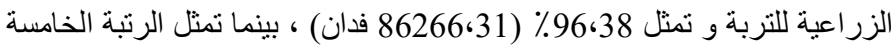

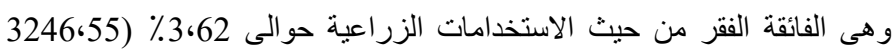

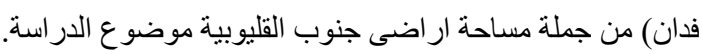

